\title{
The Maximum Displacement for Linear Probing Hashing
}

Niclas Petersson

Department of Mathematics

Uppsala University 
Dissertation presented at Uppsala University to be publicly examined in Polhemsalen, Ångströmlaboratoriet, Lägerhyddsvägen 1, Uppsala, Friday, February 27, 2009 at 13:15 for the degree of Doctor of Philosophy. The examination will be conducted in English.

\begin{abstract}
Petersson, N. (2009). The Maximum Displacement for Linear Probing Hashing. Uppsala Dissertations in Mathematics 63. viii+20 pp. Uppsala. ISBN 978-91-5062050-4.
\end{abstract}

In this thesis we study the standard probabilistic model for hashing with linear probing. The main purpose is to determine the asymptotic distribution for the maximum displacement. Depending on the ratio between the number of items and the number of cells, there are several cases to consider. Paper I solves the problem for the special case of almost full hash tables. That is, hash tables where every cell but one is occupied. Paper II completes the analysis by solving the problem for all remaining cases. That is, for every case where the number of items divided by the number of cells lies in the interval $[0,1]$.

The last two papers treat quite different topics. Paper III studies the area covered by the supremum process of Brownian motion. One of the main theorems in Paper I is expressed in terms of the Laplace transform of this area. Paper IV provides a new sufficient condition for a collection of independent random variables to be negatively associated when conditioned on their total sum. The condition applies to a collection of independent Borel-distributed random variables, which made it possible to prove a Poisson approximation that where essential for the completion of Paper II.

Keywords: Probabilistic analysis of algorithms, hashing, linear probing, negative dependence, Brownian motion.

Niclas Petersson, Department of Mathematics, Box 480, Uppsala University, SE-751 06 Uppsala, Sweden

Copyright (C) Niclas Petersson, 2009

ISSN 1401-2049

ISBN 978-91-506-2050-4

urn:nbn:se:uu:diva-9545 (http://urn.kb.se/resolve?urn=urn:nbn:se:uu:diva-9545) 
Finally, we need a great deal of faith in probability theory when we use hashing methods, since they are efficient only on the average, while the worst case is terrible!

Donald Knuth, The Art of Computer Programming 



\section{List of Papers}

This thesis is based on the following papers, which are referred to in the text by their Roman numerals.

I Petersson, N. (2008). The maximum displacement for linear probing hashing I.

II Petersson, N. (2009). The maximum displacement for linear probing hashing II.

III Janson, S. And Petersson, N. (2007). The integral of the supremum process of Brownian motion.

IV Petersson, N. (2008). Negative dependence of independent random variables conditional on their total sum. 



\section{Contents}

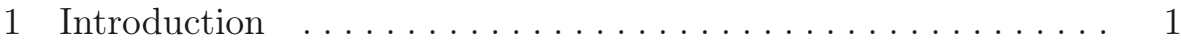

1.1 Linear Probing Hashing. . . . . . . . . . . . . . . . 1

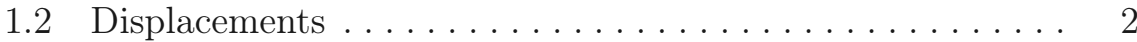

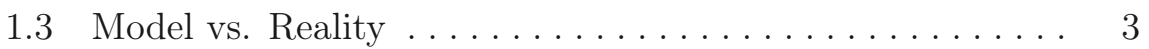

1.4 Variations of the Algorithm................ 5

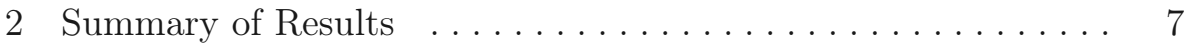

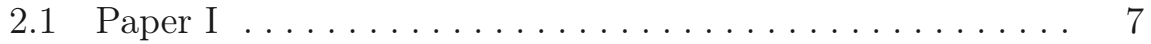

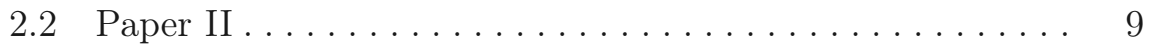

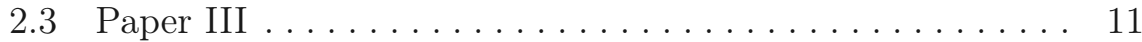

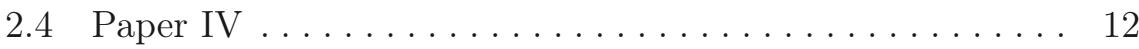

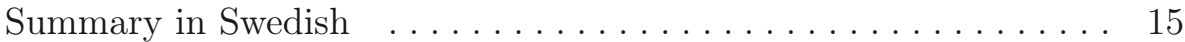

Acknowledgements ..................... 17

Bibliography ............................ 19 



\section{Introduction}

As research subject, hashing is shared by mathematicians and computer scientists with a common interest in probabilistic analysis of algorithms. Hashing can in the present context be described as a special class of search methods used for storage of information in a computer memory or database. ${ }^{1}$ Such methods are also called scatter storage techniques, since the principal idea is to spread the information randomly in the memory. Although the subject of hashing originates from computer science, it is actually quite advantageous to introduce the algorithm in a strictly mathematical way. Especially in this thesis, which is devoted to the more mathematical aspects of hashing.

\subsection{Linear Probing Hashing}

The standard version of hashing with linear probing can be described as follows, where $n$ and $m$ are integers such that $0 \leq n \leq m$. (For the classic, computer science orientated definition, see Knuth [14, Sec. 6.4, Algorithm 6.4.L].)

$n$ items $x_{1}, \ldots, x_{n}$ are placed sequentially into a table with $m$ cells $1, \ldots, m$ according to some hash sequence $\left\{h_{i}\right\}_{1}^{n}$ where $h_{i} \in\{1, \ldots, m\}$. That is, item $x_{i}$ is inserted into cell $h_{i}$ if it is empty. If not, then $x_{i}$ (linearly) probes cell $h_{i}+1, h_{i}+2$, etc until an empty cell is found and the item can be inserted. All positions in the table can be interpreted modulo $m$ as any item that is rejected from the last cell $m$, continues to probe cell 1, 2, etc until it is inserted in an empty cell.

Throughout this thesis, all hash sequences also follow the standard probabilistic model. ${ }^{2}$ That is, the hash addresses $h_{i}$ are independent random numbers, uniformly distributed on $\{1, \ldots, m\}$. Hence all $m^{n}$ possible hash sequences $\left\{h_{i}\right\}_{1}^{n}$ are equally likely when choosing a sequence at random. A hash table with $m$ cells and $n$ items generated by the above algorithm will from now on be denoted $\mathcal{T}_{m, n}$.

\footnotetext{
${ }^{1}$ The first known documentation of the main ideas behind hashing is an internal IBM memorandum by H. P. Luhn from 1953, see Knuth [14, Sec. 6.4]. The word hashing did not become standard terminology (in print at least) until the late 1960s.

${ }^{2}$ The validity of this model is briefly discussed in Section 1.3.
} 
A hash table $\mathcal{T}_{m, n}$ is called full if $n=m$ and almost full if $n=$ $m-1$. A non-full hash table is said to be confined if the last cell in the table is empty. Confined hash tables are from now on distinguished with a prime $\mathcal{T}_{m, n}^{\prime}$. Due to the circular symmetry of the construction procedure, all cells are equally likely to be empty in the final table. Thus, a confined hash table is nothing but a standard table conditioned on the last cell being empty. So any non-full hash table can be given the confined property by choosing an empty cell with uniform probability (among all the empty cells) and then relabeling the table so that the chosen cell becomes the last. It can therefore be assumed that the hash table is confined whenever it is suitable for the analysis.

A block in a hash table $\mathcal{T}_{m, n}^{\prime}$ is defined as a sequence of consecutive cells $\{i+1, \ldots, j\}$ (modulo $m$ ) such that cell $i$ and $j$ are empty and all cells in between are occupied. Thus, every block contains exactly one empty cell (the last one) and the table has as many blocks as it has empty cells. The total number of blocks (usually denoted by $N$ ) is therefore given by $N=m-n$. The smallest block size is 1 , since an empty cell following the empty cell of the previous block constitutes a block of its own. Note that the internal structure of different blocks are independent of each other. Thus, any block of a given length $k$ may be regarded as a confined almost full hash table $\mathcal{T}_{k, k-1}^{\prime}$ in itself. An almost full hash table $\mathcal{T}_{m, m-1}^{\prime}$ is in this sense the most primitive type of table, since it only consists of one block.

\subsection{Displacements}

If item $x_{i}$ is inserted into cell $q_{i}$, then its displacement or individual displacement is

$$
d_{i}:=\left(q_{i}-h_{i}\right) \bmod m .
$$

Thus, the displacement equals the number of unsuccessful probes item $x_{i}$ makes before being inserted into an empty cell. The displacement is a measure of time (or cost) it takes to insert an item into the table. Once the hash table has been made, $x_{i}$ may be allocated with the same type of linear probing as when the item was inserted. That is, by probing cell $h_{i}$, $h_{i}+1$, etc until the item is found. The search time needed to allocate $x_{i}$ in the final table is therefore proportional to $d_{i}+1$. Some displacements has already been thoroughly analyzed. The total displacement, i.e.

$$
D:=\sum_{i=1}^{n} d_{i},
$$

has for instance been studied by Knuth [14], Flajolet, Poblete and Viola [8] and Janson [9]. The (individual) displacement of an item chosen at random has also been studied in detail by Janson [10] and Viola [20]. 
The maximum displacement (or worst case) is defined as

$$
\mathfrak{D}:=\max _{1 \leq i \leq n} d_{i} .
$$

The primary purpose of this thesis is to determine the asymptotic distribution of the maximum displacement for all $0 \leq \lambda \leq 1$ when $n, m \rightarrow \infty$ in such a way that $n / m \rightarrow \lambda$. Since the maximum displacement also is the record among the maximum displacements within each block, it is important to know how the block sizes are distributed. Block behavior has been studied by Chassaing et al. ${ }^{3}$ in [3], [4] and [5] and Janson [9].

A secondary purpose is to study the number of items with displacements very close to the maximum. Such items are from now on said to have a critical displacement. The exact definition of a critical displacement must be adjusted to the ratio $\lambda$, but the principle is to define

$$
\text { "item } x_{i} \text { has a critical displacement" } \Leftrightarrow d_{i} \geq \zeta,
$$

for some appropriate function $\zeta=\zeta(m, n)$. Here, the word appropriate means that the total number of critical displacements should be a nondegenerate random variable asymptotically.

\subsection{Model vs. Reality}

The goal of this section is to connect the mathematical model for hashing to its origin in computer science. This will be done with the aid of a small example in form of the creation of an address book.

Assume that one wants to store some sort of basic contact information to $n$ different persons in a hash table with $m$ cells. Let every person be identified with a unique key formed by the contraction of that persons fore- and surname. This is a simple way to produce an key that also represents the attached information. ${ }^{4}$ The author's current contact information could for instance be;

\begin{tabular}{l|ll} 
KEY & NICLASPETERSSON & \\
\hline DAtA & Niclas Petersson & 買 $+46(0) 768171871$ \\
& S:t Olofsgatan 66A & $\mathbf{0}+46(0) 18144209$ \\
& 75330 Uppsala & $凶$ niclasp@math.uu.se \\
& Sweden & $\star$ October 9, 1977
\end{tabular}

The information above constitutes an item in the mathematical model. If the data part is small enough it is possible to store both key and data

\footnotetext{
${ }^{3}$ Co-authors: Janson [3], Louchard [4] and Marckert [5].

${ }^{4}$ In case some persons share the same fore- and surname one could add a middle name (or perhaps a number) in order to give them separate keys.
} 
in one cell. If there is a lot of data attached to some keys, one should consider to store the data elsewhere. The cell should then contain the key and a pointer to the actual place of storage instead. In the example above all items will be of approximately the same small size and it therefore makes sense to store key and data together. Thus, each cell must be of such size that it (easily) can contain the data of a person with a lot of contact information. The choice of cellsize will also determine the size of the total amount of memory that is needed for the hash table.

In order to hash a key $K_{i}$ into the table one needs some sort of hash function $h$. The hash function in this example should be able to transform a string of letters, for example

$$
K_{i}=\text { NICLASPETERSSON }
$$

into a natural number $h_{i}=h\left(K_{i}\right)$ such that $1 \leq h_{i} \leq m$. The ideal situation would be if every hash address is unique, i.e. $h_{i} \neq h_{j}$ for every $i$ and $j$ such that $1 \leq i<j \leq n$. Without collisions there are no displacements and any table look-up will therefore be performed almost instantly. Such hash functions are unfortunately virtually impossible to construct for most tables of practical importance.

If the idea of finding a hash function that can produce a sequence $\left\{h_{i}\right\}_{1}^{n}$ of $n$ unique addresses is abandoned, what should replace it? In the probabilistic model, every item (or key) is given an (independent) random number, uniformly distributed on $\{1, \ldots, m\}$, as hash address. A hash function able to produce this result would truly be great since no systematic errors would ever occur, regardless what the keys look like. In practice however, it is impossible to achieve true random numbers from strings of non-random letters. Fortunately, there exists a lot of techniques designed to simulate random behavior that works very well. ${ }^{5}$ Hash functions can also exploit non-random properties in the keys that causes fewer collisions than true random numbers would do.

The effectiveness of a computer program is very much dependent on the search methods it uses to access needed information. A significant part of the total running time for many programs are caused by searching. Hashing is (when properly used) often an effective way to enhance the performance by reducing the search times. To computing the hash address for a given key is an instant task, when the hash function has been decided. To find the address corresponding location in the memory is something that simply must be done regardless of storing method. Thus, the drawback of hashing occurs when (frequently used) data is subject to huge displacements. The size of the table and the ratio between the number of items and cells should therefore be chosen with care.

\footnotetext{
${ }^{5}$ How to construct such hash functions is beyond the scope of this thesis. The interested reader may consult Knuth [14] to learn the basics.
} 
Remark 1.1. Assume that $n<365$ so that it is possible to chose $m=365$. Instead of using the name as key one could then use the birthday (provided every persons set of data contain the date of birth). The obvious hash function would then transform the birthday into a number between 1 and 365 according to the day's place in the calender. The authors birthday would for instance generate the hash address 282 . Except for a minor bias in cell 59, this method would generate a very good approximation of a uniform probability distribution. ${ }^{6}$ The famous birthday problem asserts that if 23 persons (or more) are present in a room, the chance is over 50 percent that at least two of them share the same birthday. Moreover, if one replaces the number 23 with 41 (or 57), the risk of a birthday collision would increase to over 90 (respectively 99) percent. It shows that collisions, and thus displacements, most certainly will occur even in sparse hash tables.

\subsection{Variations of the Algorithm}

This thesis exclusively uses the standard algorithm to model linear probing hashing. However, it is easy to think of variations of the algorithm that also would be very interesting to analyze. It is for instance possible to change the (so called) insertion policy that rules how collisions are resolved during the creation of the table. For example, consider the following three insertion policies;

FC First Come (First Served). The algorithm described on page 1 where the first item that probes a cell is inserted and remains there.

LC Last Come (First Served), see Poblete and Munro [17]. Each new item is inserted where it arrives. If the cell is already occupied, the old inhabitant moves one step to the right. If that to is occupied, its old inhabitant is moved etc.

RH Robin Hood, see Celis, Larson and Munro [2]. When an item probes a cell that is already occupied by another item, the item (of the two) with the largest current displacement gets the cell. The other item is moved to the next cell where the same rule applies recursively. (Ties are resolved either way.)

The LC- and RH-policies tend to give more evenly distributed displacements than the FC-policy, see Poblete, Viola and Munro [18] and Janson [10]. The RH-policy also minimizes the variance of the (individual) displacements for all linear probing algorithms, see Carlsson, Munro and Poblete [1]. The maximum displacement for linear probing hashing with the RH-policy has been studied by Devroye, Morin and Viola [6].

\footnotetext{
${ }^{6}$ People born February 29 are assumed to have birthday February 28.
} 
It is also possible to change the probing method in the algorithm. For example, quadratic, uniform and random probing are all common alternatives to linear probing. Quadratic (or square) probing, is defined such that item $x_{i}$ probes the cells quadratically $h_{i}, h_{i+1}, h_{i+4}, h_{i+9}$, etc until an empty position is found. Thus, if position $h_{i}$ is part of a large cluster of occupied cells, it is not necessary to probe all cells in the cluster before finding an empty cell. There also exist methods such as double hashing, which uses two hash functions instead of one, and hashing with chaining, where the cells in the original table are linked lists or small hash tables in themselves. Since these methods are not treated in the thesis, they will not be described in detail. The interested reader can find some of the probing methods and algorithms above thoroughly defined in Knuth [14]. 


\section{Summary of Results}

The primary purpose of this thesis is to determine the asymptotic distribution for the maximum displacement $\mathfrak{D}$. The problem is solved in Paper I and II for all $0 \leq \lambda \leq 1$ when $n, m \rightarrow \infty$ in such a way that $n / m \rightarrow \lambda$. Although the last two papers treat quite different topics, they both contain results that where essential for the completion of the first two papers (and thus the thesis). Paper III studies the area covered by the supremum process of Brownian motion. Paper IV provides a new sufficient condition for a collection of independent random variables to be negatively associated when conditioned on their total sum.

\subsection{Paper I}

One of the main results in Paper I is the distribution of the maximum displacement in an almost full hash table. That is, the distribution of the worst displacement in the worst type of table. The result is also a starting point for further analysis since every hash table consists of a number of blocks which all are almost full hash tables in themselves.

Theorem 2.1. If $m \rightarrow \infty$, then the maximum displacement $\mathfrak{D}$ in an almost full hash table $\mathcal{T}_{m, m-1}^{\prime}$ is given by

$$
\mathbb{P}\left(\frac{m-\mathfrak{D}}{m^{2 / 3}} \leq z\right) \rightarrow 1-\psi\left(z^{3 / 2}\right), \quad 0<z<\infty,
$$

where $\psi$ is the function defined by

$$
\psi(s)=\frac{1}{\Gamma(2 / 3)} \sum_{n=0}^{\infty} \frac{\Gamma(n+2 / 3)}{\Gamma(3 n / 2+1)}\left(\frac{-3 \sqrt{2} s}{4}\right)^{n} .
$$

The proof of Theorem 2.1 is based on the idea of reversing the time direction for the hashing procedure. Consider a confined almost full random hash table $\mathcal{T}_{m, m-1}^{\prime}$ constructed by some unknown hash sequence $\left\{h_{i}\right\}_{i=1}^{m-1}$, where each $h_{i}$ where chosen with uniform probability. By removing the items one by one in reversed sequential order, the evolution of the table can be reconstructed in an algorithmic way. The largest block is then subject to a fragmentation as its size will decrease when items are removed from the table. Thus, the probability for items to 
have a large displacement decreases as the largest block is fragmented. At some point the size of the largest block will be less than the maximum displacement found so far. The fragmentation can then be stopped since no remaining item is capable of beating the current maximum.

Let $r(m)=o(\sqrt{m})$ be an arbitrary increasing function of $m$ and let the number $N$ of empty cells in $\mathcal{T}_{m, m-N}^{\prime}$ be a function of time and $r(m)$,

$$
N=N_{t}^{m}=\lceil\operatorname{tr}(m)\rceil, \quad t>0 .
$$

The size of the largest block, which is denoted by $\eta$, then becomes a continuous time process,

$$
\eta=\eta_{t}^{m}=\eta(m, m-\lceil t r(m)\rceil), \quad t>0 .
$$

Let $X_{t}^{m}$ be the normalized process of $\eta_{t}^{m}$ defined by

$$
X_{t}^{m}=\frac{m-\eta_{t}^{m}}{r(m)^{2}}, \quad t>0 .
$$

A fundamental result in Paper I is that $\left\{X_{t}^{m}\right\}$ converge to the Lévy process known as the stable subordinator of index $1 / 2$. If $X_{t}$ denotes the subordinator, then for any fixed $t>0$,

$$
\mathbb{P}\left(X_{t} \leq x\right)=\frac{1}{\sqrt{2 \pi}} \int_{0}^{x / t^{2}} y^{-3 / 2} e^{-1 / 2 y} \mathrm{~d} y .
$$

Theorem 2.2. For any normalizing sequence $r(m)=o(\sqrt{m})$, the sequence of random processes $\left\{X_{t}^{m}\right\}$ converges in distribution to the Lévy process known as the stable subordinator of index $1 / 2$,

$$
X_{t}^{m} \stackrel{\mathrm{d}}{\longrightarrow} X_{t} \quad \text { as } \quad m \rightarrow \infty,
$$

in the usual Skorohod topology on $\mathcal{D}[0, \infty)$.

That $\left\{X_{t}^{m}\right\}$ converge to $X_{t}$ for fixed time-points has been known for some time. It follows from the fact that there is a one-to-one correspondence between linear probing hash tables and Pavlov's random forests. If $\left(\nu_{1}, \ldots, \nu_{N}\right)$ denotes the block sizes in $\mathcal{T}_{m, n}^{\prime}$ and $\left(T_{1}, \ldots, T_{N}\right)$ denotes the tree sizes in a random forest with $n$ non-root vertices and $N$ roots, then

$$
\left(\nu_{1}, \ldots, \nu_{N}\right) \stackrel{\mathrm{d}}{=}\left(T_{1}, \ldots, T_{N}\right) \stackrel{\mathrm{d}}{=}\left(B_{1}, \ldots, B_{N} \mid B^{(N)}=N+n\right),
$$

where $B_{1}, \ldots, B_{N}$ are independent Borel-distributed random variables, see Knuth [14, Sec. 6.4, Exercise 31], Chassaing and Louchard [4, Sec. 8] and Janson [9, Theorem 4.1]. In particular, the largest block in the hash table has the same distribution as the largest tree in the random forest. Pavlov's results for the size of the maximum tree in a random forest can therefore be applied to hash tables as well, see [16, Chap. 2]. 


\subsection{Paper II}

Paper II extends the analysis initiated in Paper I to include all cases where $n<m$. Recall that $N=m-n$ is the number of empty cells in $\mathcal{T}_{m, n}^{\prime}$ and let $n, N \rightarrow \infty$ in such a way that $\lambda_{N}=n /(n+N) \rightarrow \lambda$ for some constant $0 \leq \lambda \leq 1$. Depending on the limit $\lambda$, a hash table is classified as sparse $(\lambda=0)$, balanced $(0<\lambda<1)$ or dense $(\lambda=1)$. In dense tables the rate of convergence also plays a fundamental role for the limit distribution of the maximum displacement. Dense tables are therefore sub-classified as barely dense, (plain) dense, very dense or ultra dense according to the following table.

\begin{tabular}{clcccc}
$\lambda$ & classification & $n / N \rightarrow$ & $n /(N \ln N) \rightarrow$ & $n / N^{2} \rightarrow$ & $n / N^{3} \rightarrow$ \\
\hline 0 & sparse & 0 & 0 & 0 & 0 \\
$(0,1)$ & balanced & $\alpha$ & 0 & 0 & 0 \\
1 & barely dense & $\infty$ & 0 & 0 & 0 \\
1 & phase transition & $\infty$ & $\alpha$ & 0 & 0 \\
1 & dense & $\infty$ & $\infty$ & 0 & 0 \\
1 & phase transition & $\infty$ & $\infty$ & $\alpha$ & 0 \\
1 & very dense & $\infty$ & $\infty$ & $\infty$ & 0 \\
1 & phase transition & $\infty$ & $\infty$ & $\infty$ & $\alpha$ \\
1 & ultra dense & $\infty$ & $\infty$ & $\infty$ & $\infty$
\end{tabular}

Two different methods are used to analyze the cases above. One method for sparse, balanced and barely dense tables and another for (plain) dense, very dense and ultra dense tables. The first method uses that the number of critical displacements converge to a Poisson distributed random variable (when chosen properly). The second method is based on the fact that the largest block is so big in really dense tables that it is very unlikely to find the maximum displacement somewhere else. That is, the largest block is of the same order as the table itself. The main results are listed below.

Theorem 2.3 (Sparse tables). Let $n, N \rightarrow \infty$ in such a way that $n / N \rightarrow 0$. Then for every $0 \leq z<\infty$,

$$
\mathbb{P}(\mathfrak{D}=r-1) \rightarrow e^{-z}, \quad \mathbb{P}(\mathfrak{D}=r) \rightarrow 1-e^{-z},
$$

where $r=r(z, n, N)$ either is a fixed natural number such that

$$
N \frac{r^{r-2}}{r !} \lambda_{N}^{r} \rightarrow \infty, \quad N \frac{(r+1)^{r-1}}{(r+1) !} \lambda_{N}^{r+1} \rightarrow z,
$$

or $r \rightarrow \infty$ in such a way that

$$
\frac{1}{\sqrt{2 \pi}} N r^{-5 / 2}\left(\lambda_{N} e^{1-\lambda_{N}}\right)^{r} \rightarrow \infty, \quad \frac{1}{\sqrt{2 \pi}} N r^{-5 / 2}\left(\lambda_{N} e^{1-\lambda_{N}}\right)^{r+1} \rightarrow z .
$$


Theorem 2.4 (Balanced tables). Let $n, N \rightarrow \infty$ in such a way that $\lambda_{N}=n /(n+N) \rightarrow \lambda$ for some constant $0<\lambda<1$. Then for every $\delta>0$ there exist constants $C_{\delta}, n_{0}$ and $N_{0}$ such that

$$
\mathbb{P}\left(\left|\mathfrak{D}-r_{0}\right| \leq C_{\delta}\right)>1-\delta \quad \text { for every pair } \quad(n, N) \geq\left(n_{0}, N_{0}\right),
$$

where $r_{0}=r_{0}(n, N)$ is defined as

$$
r_{0}=\beta^{-1}\left(\ln N-\frac{5}{2} \ln \ln N\right)
$$

and where $\beta=\beta(n, N)$ in turn is defined as

$$
\beta=-\ln \left(\lambda_{N} e^{1-\lambda_{N}}\right) .
$$

Theorem 2.5 (Barely dense tables). Let $n, N \rightarrow \infty$ in such a way that $n / N \rightarrow \infty$ and $n /(N \ln N) \rightarrow 0$. Then for every $-\infty<z<\infty$,

$$
\mathbb{P}(\mathfrak{D}<r) \rightarrow \exp \left\{-e^{-z}\right\},
$$

where $r=r(z, n, N)$ run through the natural numbers in such a way that

$$
r=\beta^{-1}\left(\ln N-\frac{5}{2} \ln \ln N-\ln 2 \sqrt{\pi}+z+o(1)\right)
$$

as $n$ and $N$ tend to infinity. Similar to the balanced case, $\beta=\beta(n, N)$ is defined as

$$
\beta=-\ln \left(\lambda_{N} e^{1-\lambda_{N}}\right)=\frac{N^{2}}{2 n^{2}}(1+O(N / n)) .
$$

Theorem 2.6 (Plain dense tables). Let $n, N \rightarrow \infty$ in such a way that $n /(N \ln N) \rightarrow \infty$ and $n / N^{2} \rightarrow 0$. Then for every $-\infty<z<\infty$,

$$
\mathbb{P}(\mathfrak{D}<r) \rightarrow \exp \left\{-e^{-z}\right\},
$$

where $r=r(z, n, N)$ run through the natural numbers in such a way that

$$
r=2\left(\frac{n}{N}\right)^{2}\left(\ln \frac{N^{2}}{n}-\frac{3}{2} \ln \ln \frac{N^{2}}{n}-\ln 2 \sqrt{\pi}+z+o(1)\right),
$$

as $n$ and $N$ tend to infinity.

Theorem 2.7 (Very dense tables). Let $n, N \rightarrow \infty$ in such a way that $n / N^{2} \rightarrow \infty$ and $n / N^{3} \rightarrow 0$. Then for every $0<z<\infty$,

$$
\mathbb{P}\left(\frac{n-\mathfrak{D}}{N^{2}} \leq z\right) \rightarrow \frac{1}{\sqrt{2 \pi}} \int_{0}^{z} y^{-3 / 2} e^{-1 / 2 y} \mathrm{~d} y .
$$

Theorem 2.8 (Ultra dense tables). Let $n \rightarrow \infty$ (and possibly $N \rightarrow$ $\infty)$ in such a way that $n / N^{3} \rightarrow \infty$. Then for every $0<z<\infty$,

$$
\mathbb{P}\left(\frac{n-\mathfrak{D}}{n^{2 / 3}} \leq z\right) \rightarrow 1-\frac{1}{\Gamma(2 / 3)} \sum_{n=0}^{\infty} \frac{\Gamma(n+2 / 3)}{\Gamma(3 n / 2+1)}\left(\frac{-3 \sqrt{2} z^{3 / 2}}{4}\right)^{n} .
$$




\subsection{Paper III}

Paper III deals with the integral of the supremum process of Brownian motion. The area covered by this integral, denoted by $\mathcal{A}$ when $(0,1]$ is the interval of integration, is formally defined as

$$
\mathcal{A}:=\int_{0}^{1} \max _{0 \leq s \leq t} B(s) \mathrm{d} t
$$

where $B(t), t \geq 0$, is (standard) Brownian motion. By Brownian scaling it is possible to show the following formula for different intervals,

$$
\mathcal{A}(T):=\int_{0}^{T} \max _{0 \leq s \leq t} B(s) \mathrm{d} t \stackrel{\mathrm{d}}{=} T^{3 / 2} \mathcal{A} .
$$

Thus, it is enough to consider $\mathcal{A}$ in any analysis.

The main theorem in Paper III gives a formula for the double Laplace transform of $\mathcal{A}$. That is, the Laplace transform of the Laplace transform. The formula is intriguing since one can use it to determine the more elusive (ordinary) Laplace transform of $\mathcal{A}$. (To determine the ordinary transform by direct calculation seems very hard, if it is even possible.)

Theorem 2.9. Let $\psi$ be the Laplace transform of $\mathcal{A}$. For all $\alpha, \lambda>0$,

$$
\int_{0}^{\infty} \psi\left(\alpha s^{3 / 2}\right) e^{-\lambda s} \mathrm{~d} s=\int_{0}^{\infty}\left(1+\frac{3 \alpha s}{2 \sqrt{2 \lambda}}\right)^{-2 / 3} e^{-\lambda s} \mathrm{~d} s .
$$

The proof of Theorem 2.9 is based on excursion theory and local time for Brownian motion. Moments of $\mathcal{A}$ can be obtained from the formula by repeated differentiation followed by some minor manipulations.

Theorem 2.10. The n:th moment of $\mathcal{A}$ is

$$
\mathbb{E} \mathcal{A}^{n}=\frac{n ! \Gamma(n+2 / 3)}{\Gamma(2 / 3) \Gamma(3 n / 2+1)}\left(\frac{3 \sqrt{2}}{4}\right)^{n}, \quad n \in \mathbb{N} .
$$

The following corollary is an immediate consequence of Theorem 2.10.

Corollary 2.11. The Laplace transform of $\mathcal{A}$ is

$$
\psi(s)=\frac{1}{\Gamma(2 / 3)} \sum_{n=0}^{\infty} \frac{\Gamma(n+2 / 3)}{\Gamma(3 n / 2+1)}\left(\frac{-3 \sqrt{2} s}{4}\right)^{n} .
$$

$\mathcal{A}$ appear in Paper I as a random Poisson-parameter. The papers main result, i.e. the distribution for the maximum displacement in an almost full hash table, is also expressed in terms of the Laplace transform of $\mathcal{A}$. 


\subsection{Paper IV}

The main result of Paper IV is a new sufficient condition for a collection of independent random variables to be negatively associated when conditioned on their total sum. The concept of negative association was introduced by Joag-Dev and Proschan [12] and is one (of several) ways to classify mutual negative dependence between random variables. A collection of random variables $\left\{X_{\alpha}\right\}$ is defined to be negatively associated if, for all disjoint sets $A_{1}, A_{2}$ of indices and all bounded functions $f$ and $g$ increasing in every variable,

$\mathbb{E}\left[f\left(X_{\alpha}, \alpha \in A_{1}\right) g\left(X_{\alpha}, \alpha \in A_{2}\right)\right] \leq \mathbb{E}\left[f\left(X_{\alpha}, \alpha \in A_{1}\right)\right] \mathbb{E}\left[g\left(X_{\alpha}, \alpha \in A_{2}\right)\right]$.

Let $\left(X_{1}, \ldots, X_{N}\right)$ be a collection of independent non-negative integervalued random variables. A combination of Theorem 2.6 in [12] and Strassen's theorem [19, Theorem 11] then gives the following result.

Theorem 2.12 (Joag-Dev and Proschan 1983; Strassen 1964). Let $X_{1}, \ldots, X_{N}$ be independent random variables such that $\left(X_{1}, \ldots, X_{N}\right)$ is stochastically increasing in $X_{1}+\cdots+X_{N}$. Then the distribution of $\left(X_{1}, \ldots, X_{N}\right)$ conditional on $X_{1}+\cdots+X_{N}$ is negatively associated.

There are at least two known sufficient conditions for a collection of independent random variables to be stochastically increasing in their total sum. One condition that was proved by Efron [7] and one that was proved by Liggett [15].

Theorem 2.13 (Efron 1965). A collection of independent random variables $\left(X_{1}, \ldots, X_{N}\right)$ is stochastically increasing in $X_{1}+\cdots+X_{N}$ if

$$
\frac{\mathbb{P}\left(X_{i}=k+1\right)}{\mathbb{P}\left(X_{i}=k\right)} \downarrow \quad \text { in } k \text { for each } i=1, \ldots, N .
$$

Theorem 2.14 (Liggett 1999). Let $X_{1}, \ldots, X_{N}$ be independent random variables and define the partial sums $S_{i}:=\sum_{j=1}^{i} X_{j}$ for $i=1, \ldots, N$. If

$$
\mathbb{P}\left(X_{i}=k+1 \mid S_{i}=n+1\right) \leq \mathbb{P}\left(X_{i}=k \mid S_{i}=n\right)
$$

and

$$
\mathbb{P}\left(S_{i-1}=k+1 \mid S_{i}=n+1\right) \leq \mathbb{P}\left(S_{i-1}=k \mid S_{i}=n\right)
$$

for every $i=2, \ldots, N$ and for every $k, n \in \mathbb{N}$ such that $k \leq n$, then $\left(X_{1}, \ldots, X_{N}\right)$ is stochastically increasing in $S_{N}$.

Efron's condition states that any collection of independent log-concave random variables is stochastically increasing in their total sum. Liggett's condition is more complex since it both allow and requires a certain degree of log-convexity among the variables. In this sense, the two conditions complement each other. The new condition looks very similar to Liggett's, but it is more general and it can be shown that both Theorem 2.13 and 2.14 are special cases of Theorem 2.15. 
Theorem 2.15. Let $X_{1}, \ldots, X_{N}$ be independent random variables and define the partial sums $S_{i}:=\sum_{j=1}^{i} X_{j}$ for $i=1, \ldots, N$. If

$$
\mathbb{P}\left(X_{i} \leq k \mid S_{i}=n\right) \leq \mathbb{P}\left(X_{i} \leq k+1 \mid S_{i}=n+1\right)
$$

and

$$
\mathbb{P}\left(S_{i-1} \leq k \mid S_{i}=n\right) \leq \mathbb{P}\left(S_{i-1} \leq k+1 \mid S_{i}=n+1\right)
$$

for every $i=2, \ldots, N$ and for every $k, n \in \mathbb{N}$ such that $k \leq n$, then $X_{1}, \ldots, X_{N}$ are stochastically increasing in $S_{N}$.

With the aid of Theorem 2.15 it was possible to prove the following result for the Borel distribution.

Theorem 2.16. Let $Y_{1}, \ldots, Y_{N}$ be independent $\operatorname{Bo}(\lambda)$-distributed random variables for some $0<\lambda<1$. That is,

$$
\mathbb{P}\left(Y_{i}=k\right)=\frac{k^{k-1}}{k !} \cdot \lambda^{k-1} e^{-\lambda k}, \quad k=1,2, \ldots
$$

for every $i=1, \ldots, N$. Then $\left(Y_{1}, \ldots, Y_{N}\right)$ is stochastically increasing in $Y_{1}+\cdots+Y_{N}$.

The result had a large impact on the sparse, balanced and barely dense case in Paper II. The random variables describing the number of critical displacements in these cases could all be Poisson approximated due to Theorem 2.16. 



\section{Summary in Swedish}

Titeln på denna avhandling kan fritt översättas till Den maximala förskjutningen för hashning med linjär sondering. Termen hashning utgör ett samlingsbegrepp för en speciell klass av probabilistiska sökmetoder som används för lagring av information i ett datorminne eller en databas. Den matematiska standardmodellen för hashning med linjär sondering kan beskrivas på följande sätt, där $n$ and $m$ är heltal sådana att $0 \leq n \leq m$, se Knuth [14, Sec. 6.4, Algorithm 6.4.L].

$n$ objekt $x_{1}, \ldots, x_{n}$ placeras $\mathrm{i}$ en tabell bestående av totalt $m$ celler $1, \ldots, m$ enligt någon hashsekvens $\left\{h_{i}\right\}_{1}^{n}$ där $h_{i} \in$ $\{1, \ldots, m\}$. Det vill säga, objekt $x_{i}$ placeras i cell $h_{i}$ förutsatt att den är tom. Om cell $h_{i}$ inte är tom sonderar $x_{i}$ (linjärt) cell $h_{i}+1, h_{i}+2$, osv tills en tom cell är funnen och objektet kan införas i tabellen. Alla positioner i tabellen kan tolkas modulo $m$ då varje objekt som avvisas från cell $m$ fortsätter att sondera cell 1, 2, osv tills den sätts in i en tom cell.

Hashsekvenserna antas dessutom följa den probabilistiska standardmodellen, dvs varje hashadress $h_{i}$ är ett oberoende slumptal, likformigt fördelat på $\{1, \ldots, m\}$. Samtliga $m^{n}$ möjliga hashsekvenser $\left\{h_{i}\right\}_{1}^{n}$ är således lika sannolika när en hashsekvens väljs på måfå.

Om objekt $x_{i}$ sätts in i cell $q_{i}$ definieras dess förskjutning som

$$
d_{i}:=\left(q_{i}-h_{i}\right) \bmod m .
$$

Förskjutningen är med andra ord en stokastisk variabel som beskriver antalet ockuperade celler som måste sonderas innan en tom cell slutligen hittas. Förskjutningen är även ett mått på tiden (eller kostnaden) som krävs för att placera objektet i tabellen. När hashtabellen väl är skapad kan objekt $x_{i}$ lokaliseras via sin ursprungsadress $h_{i}$. Även det sker med linjär sondering av $h_{i}, h_{i}+1$, etc tills objektet är återfunnet. Söktiden som krävs för lokaliseringen är därmed proportionell mot $d_{i}+1$.

Hashtabellens maximala förskjutning definieras som

$$
\mathfrak{D}:=\max _{1 \leq i \leq n} d_{i} .
$$

Huvudsyftet med avhandlingen är att bestämma $\mathfrak{D}: s$ asymptotiska fördelning för alla $0 \leq \lambda \leq 1$ när $n, m \rightarrow \infty$ så att $n / m \rightarrow \lambda$. Artikel I behandlar specialfallet $n=m-1$ och artikel II behandlar övriga fall. 
Artikel III och IV behandlar inte hashning med linjär sondering explicit. Däremot används vissa resultat i de både artiklarna för att lösa delar av avhandlingens huvudproblem. I artikel III studeras integralen av supremumprocessen för Brownsk rörelse. Den area som täcks av ovan nämnda integral förekommer i artikel I i form av en (stokastisk) Poisson parameter. I artikel IV presenteras ett nytt tillräckligt villkor för att en samling av oberoende stokastiska variabler ska vara negativt associerade betingat på deras totala summa. Villkoret går att applicera på en samling oberoende Borel-fördelade stokastiska variabler, vilket underlättade analysen av den maximala förskjutning i artikel II. 


\section{Acknowledgements}

Many people have encouraged and supported me during my time as a $\mathrm{PhD}$ student. I especially want to thank the following persons.

Svante Janson, your importance can not be overestimated. Sure, I would recommend you as advisor for your proof-reading skills alone, but what I have appreciated most is learning new mathematics from you. Several times when I thought my research problem was a dragon, impossible to slay, you provided me with a new sharp sword to fight it with. My deepest thanks!

My family, I know there where times when you would have liked to roll up your sleeves and finish the dragon for me. The fact that you always provided me with strong moral support instead (and helped me with a lot of practical things) ${ }^{1}$ is very appreciated. Thank you!

My friends, I think it is fair to say that you all helped me in your own special way. You really made a difference. I feel particularly indebted to Johan Dasht, Norbert Euler and Henrik Wanntorp for their great friendship in and outside the mathematical community.

Finally, I also owe a lot of gratitude to FMB, the (Swedish) Graduate School in Mathematics and Computing, for excellent financial support.

\footnotetext{
${ }^{1}$ Like buying your own Christmas gift and signing the card "Från Niclas" when you knew I was to busy writing this thesis to have time to go shopping.
} 



\section{Bibliography}

[1] Carlsson, S., Munro, J. I. and Poblete, P. V. (1987). On linear probing hashing. Unpublished manuscript.

[2] Celis, P., Larson, P-Å. and Munro, J. I. (1985). Robin Hood hashing. FOCS 26, 281-288.

[3] Chassaing, P. and Janson, S. (2001). A Vervaat-like path transformation for the reflected Brownian bridge conditioned on its local time at 0. Ann. Probab. 29, no. 4, 1755-1779.

[4] Chassaing, P. and Louchard, G. (2002). Phase transition for parking blocks, Brownian excursion and coalescence. Rand. Struct. Alg. 21, no. 1, 76-119.

[5] Chassaing P. and Marckert, J-F. (2001). Parking functions, empirical processes, and the width of rooted labeled trees. Electron. J. Combin. 8, no. 1, \#R14.

[6] Devroye, L., Morin, P. And Viola, A. (2004). On worst-case Robin Hood hashing. SIAM. J. Comput. 33, no. 4, 923-936.

[7] Efron, B. (1965). Increasing properties of Pólya frequency functions. Ann. Math. Stat. 36, no. 1, 272-279.

[8] Flajolet, P., Poblete, P. And Viola, A. (1998). On the analysis of linear probing hashing. Algorithmica 22, no. 4, 490-515.

[9] Janson, S. (2001). Asymptotic distribution for the cost of linear probing hashing. Random Struct. Alg. 19, no. 3-4, 438-471.

[10] Janson, S. (2005). Individual displacements for linear probing hashing with different insertion policies. ACM Transactions on Algorithms 1, no. 2, 177213.

[11] Janson, S., Łuczak, T. And Rucinski, A. (2000). Random graphs. 1st ed., Wiley-Interscience, New York,

[12] Joag-Dev, K. and Proschan, F. (1983). Negative association of random variables with applications. Ann. Statist. 11, no. 1, 286-295.

[13] Karatzas, I. And Shreve, S. E. (2004). Brownian Motion and Stochastic Calculus. 2nd ed., Springer, New York.

[14] Knuth, D. E. (1998). The Art of Computer Programming. Vol. 3: Sorting and Searching. 2nd ed., Addison-Wesley, Reading, Mass.

[15] Liggett, T. M. (2000). Monotonicity of conditional distributions and growth models on trees. Annals of Probability. 28, no. 4, 1645-1665. 
[16] Pavlov, Y. L. (2000). Random Forests. 1st ed., VSP BV, Ridderprint bv, Ridderkerk.

[17] Poblete, P. V. And Munro, J. I. (1989). Last-come-first-served hashing. J. Algorithms 10, no. 2, 228-248.

[18] Poblete, P. V., Viola, A. and Munro, J. I. (1997). The diagonal Poisson transform and its application to the analysis of a hashing scheme. Rand. Struct. Alg. 10, no. 1-2, 221-255.

[19] Strassen, V. (1965). The existence of probability measures with given marginals. Ann. Math. Statist. 36, no. 2, 423-439.

[20] Viola, A. (2005). Exact distributions of individual displacements in linear probing hashing. ACM Transactions on Algorithms, 1, no. 2, 214-242. 